\title{
Comparative Analysis of Angle Based and Traditional Routing Protocols for MANETs / WSNs
}

\author{
Saurabh mishra \\ Department of ECE \\ DIT University \\ Dehradun, India \\ Email: saurabh.mishra@dituniversity.edu.in
}

\author{
Dr. Sandip Vijay \\ Dean, Faculty of Science and Technology \\ ICFAI University \\ Dehradun, India \\ Email: vijaysandip@gmail.com
}

\begin{abstract}
In this paper Angle based routing protocols are discussed and analyzed, which are developed for Mobile AdHoc networks or mobile Wireless Sensor Networks. Moto of this paper is to provide an idea that the routing protocols developed for Mobile Adhoc Networks can be completely used in Mobile Wireless Sensor Networks, by applying angle-based approach with them, as described in AODV, DYMO, OLSR, ZRP. This paper presents the approach of angle based routing protocol which are compared with the traditional routing protocols. The protocols are simulated on Qualnet simulator. The results shows different parameters of routing protocols like packet sent, packet received and forwarded at MAC layer etc.
\end{abstract}

Index Terms-Angle based protocol; RREQ; WSNs;.

\section{IntRODUCTION}

Routing of the information is a dominant issue in designing of wireless sensor networks, due to limited resources of energy, processing power, and memory. This study proves that it requires an urgent requirement to design energy efficient routing protocols for wireless sensor networks for increasing the network lifetime [1]. To minimize the required energy consumption of the wireless sensor network, many types of the routing protocols and routing algorithms have been projected so far all around the world. The standard existence of a wireless sensor network can be enlarged extensively if the operating system of network, the application layer of the model and the network routing protocols are designed to be energy conscious. These routing protocols and algorithms must have to be awake of the sensor network hardware and must be able to use special characteristics of the micro-processors based components and transmitter and receiver terminals to minimize the wireless sensor node's energy utilization. This may move forward to a traditional solution for different types of protocol designing. For the same angle based approach is incorporated in the traditional routing protocols for Mobile Adhoc Networks MANETs to get them utilized for Wireless Sensor Networks also. This may also guide to the different types of mutual algorithms in wireless sensor networks arena. [2].

\section{Angle Based Approach}

In this work angle-based mechanism, which was proposed in [3] is utilized. This approach utilizes the geological information of a nearby mobile sensor node in the sensor network and immobile sink node to decrease the number of RREQ (route request) packets. It is understood that every sensor node consists of a GPS system inbuilt in it to recognize its geological spot and also consists of information about immobile sensor node position.

The fundamental idea of the angle-based approach is that as a sensor node which is not a sink node receives a route request RREQ packet, then the sensor node measures the angle between the node which has sent the route request RREQ, and the receiver node itself by utilizing their geological location information. If the measured angle is larger than the threshold angle then the receiver node just drops the route request RREQ packet; or else, it broadcasts the route request packet RREQ again. The basis for plummeting a route request RREQ packet that does not satisfy the above principle is that the sensor node, which is exterior of the threshold angle, hardly finds the shortest path to the sink node since the node's geological distance to the sink node is farther than that of the route request RREQ sending node.

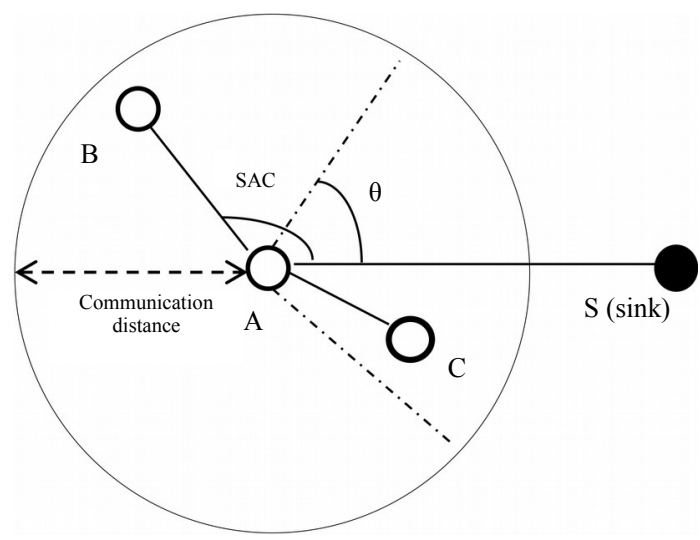

Fig. 1. Basic Idea of Angle-based mechanisms.

Fig.1. shows the fundamental concept of angle-based approach [3]. In this figure, node A broadcasts a route request RREQ packet to discover paths to a sink node. The node $B$ accept the route request packet and measures the angle based on the geological position of the sending node $\mathrm{A}$ and its own. Node B drops the route request packet since the angle $\mathrm{SAB}$, is larger than the threshold angle $\theta$. When node $\mathrm{C}$ receives the packet it also measures the angle based on the geological position of the sending node $\mathrm{A}$ and its 
own, it broadcasts the route request RREQ packet again because its angle SAC, is smaller than threshold angle $\theta$.

Main deciding factor for packet broadcast and packet drop is the threshold angle. So, here is the formula for calculating threshold angle:

$\theta=\theta_{\text {init }}-\alpha \times$ speed $^{\text {send } \_ \text {node }}-\beta \frac{\text { Number of sensor nodes }}{\text { Size of sensing area }}$

Where, $\alpha$ and $\beta=$ constants

received

$\theta_{\text {init }}=$ initial threshold angle as he route request is sending node

speed $^{\text {send_node }}=$ speed of route request RREQ

The above equation provides a small value of threshold angle to a sensor node which is located in a area where sensor deployed density is high and if the route request RREQ packet is received from a node of greater speed [3]. The reason behind is that because an when the node sending route request RREQ packet has higher speed, it possibly moves away out from the original location from where the node initiated the route request RREQ packet; so, the RREQ sending node has larger likelihood of failing to receive the RREQ packet returning from a sink node and the likelihood can be reduced by giving a smaller threshold angle. The threshold angle calculation is adjusted according to the density of sensor deployments in a area because if more sensor nodes be present in an area, then more routes can be recognized to a sink node, and so even if the threshold angle is getting smaller, the node can still find paths to a sink.

Following routing protocols have been simulated and analyzed with the angle based approach in the qualnet simulator.

\section{A. Ad hoc On Demand Distance Vector (AODV)}

Adhoc On-Demand Distance Vector AODV is a type of 'on demand routing protocol' with a minute wait-time. It means that nodes create the routes only when it is required to decrease traffic overhead. AODV protocol is compatible with Unicast, Broadcast and Multicast. In AODV protocol a routing table is developed and expanded by a particular order number to every destination node and by a time to live for every entry. It is also expanded by routing flags, the interface, a list of precursors and for outdated routes the last hop count is stored [5].

\section{B. Dynamic MANET On-demand Routing Protocol} (DYMO)

Dynamic Mobile Adhoc Networks On-demand routing Protocol (DYMO) is a descendant of the AODV routing protocol. It operates almost same to that of AODV. DYMO is the simplified version of AODV and it retain the basic mode of operation of AODV. As is the case with all table driven ad hoc routing protocols, DYMO also utilizes two protocol operations: route discovery and route maintenance [5].

\section{Optimized Link State Routing (OLSR)}

Optimized Link State Routing is a pro-active protocol, which is also based on table-driven in nature. It utilizes the link-state approach in an optimized way to diffuse topology knowledge. Typically, link-state algorithm circulates linkstate information throughout the sensor network [6].

\section{Zone Routing Protocol (ZRP)}

The Zone Routing Protocol (ZRP) is a hybrid of the proactive and reactive mechanism. It maintains an state-ofthe-art topological plot of a zone centred on every sensor node. The routes are immediately available if desired destination is within the zone. If the destinations are outside the zone, ZRP employs a route discovery procedure, which can be benefited from the local routing information of different zones $[7,8]$.

\section{SIMULATION TOOL}

In this research work for comparison of traditional and proposed angle based mechanism protocol, we have utilized QualNet 5.0.2 for the simulations and analysis of wireless sensor network. QualNet is a developer for network evaluation software that analyzes the performance of wired, wireless and hybrid network protocols and device models, useful for simulating diverse types of networks. QualNet supports thousands of nodes for simulation and also supports for 64 bit Operating system [4].

Qualnet has been used to simulate various robust models of wireless sensor networks with almost 50,000 sensor nodes [4].

\section{SIMULATION ENVIRONMENT}

In the scenario 61 nodes of network, 60 nodes are connected with one node, where node 61 is static i.e. there will be no movement in this node and node 1-60 will have random waypoint mobility model and node 61 is a full function device and work as a PAN coordinator and other nodes 1-60 are reduced function device in sensor network. The scenarios area is $1500 \mathrm{~m} * 1500 \mathrm{~m}$. and mobility model is random waypoint with mobility of $10 \mathrm{mbps}$.

In QualNet Simulator, following parameters are configured before simulation. Table 1 shows these parameters along with their values.

TABLE I. SIMULATION PARAMETERS

\begin{tabular}{|l|l|l|}
\hline S.No. & \multicolumn{1}{|c|}{ Parameters } & \multicolumn{1}{c|}{ Values } \\
\hline 1 & Simulation Time & $100 \mathrm{sec}$ \\
\hline 2 & Mobility Model & $\begin{array}{l}\text { Random way Point for sensor } \\
\text { nodes, None for sink nodes }\end{array}$ \\
\hline 3 & Simulation Area & $1500 * 1500 \mathrm{~m}^{2}$ \\
\hline 4 & Radio Type & 802.15 .4 \\
\hline 6 & MAC Protocol & 802.15 .4 \\
\hline 7 & Number of Nodes & 61 \\
\hline
\end{tabular}


Scenarios and simulation for both the networks are shown below:-

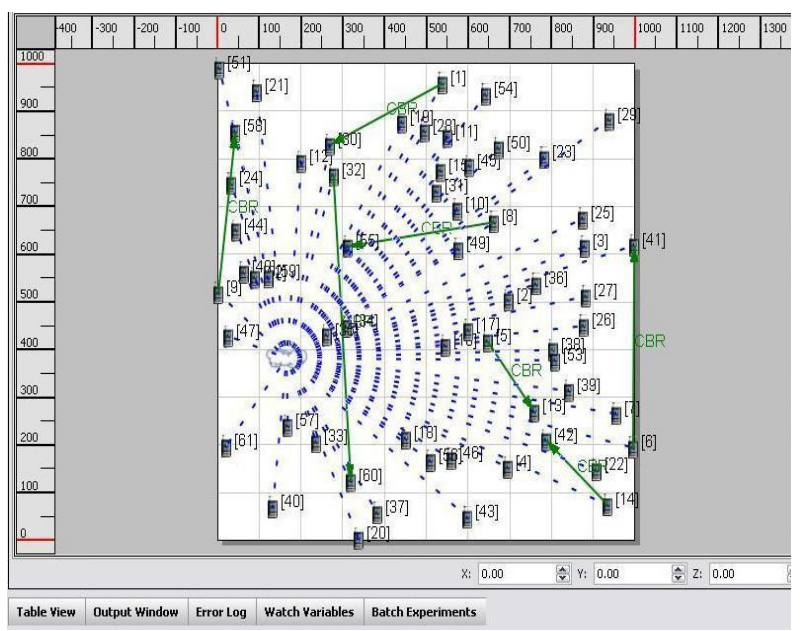

Fig. 2. Scenerios for WSN.

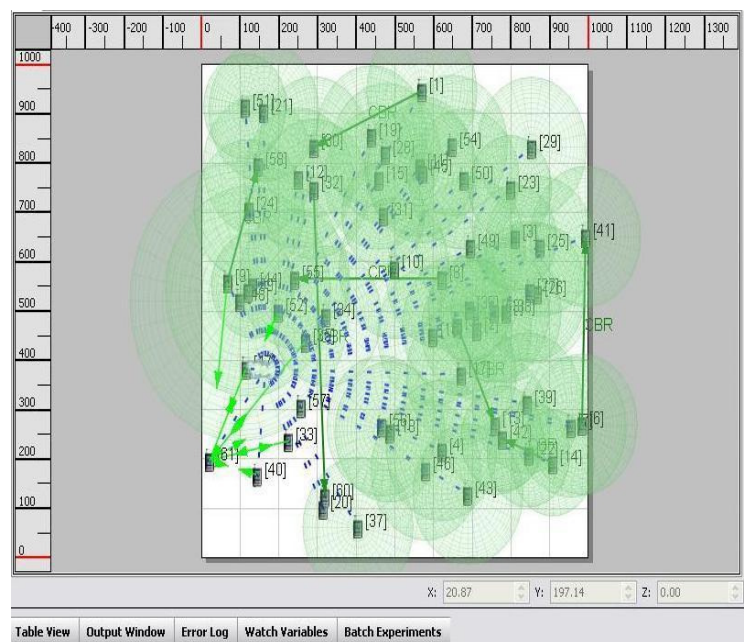

Fig. 3. Simulation for WSN.

\section{RESULTS AND DisCUSSIONS}

Using QualNet 5.0.2 simulator different parameters for both networks are analyzed. Results of analysis are as follows:-

\section{A. Number of data request received}

Fig. 4 shows graph of typical and modified protocols with respect to number of data request received. Number of data request received in protocols is varied except in ZRP, whereas major difference can be seen AODV \& AAODV. DYMO \& ADYMO has slight difference in values. In AOLSR, it can be clearly seen that data packet are decreased as compared to OLSR.

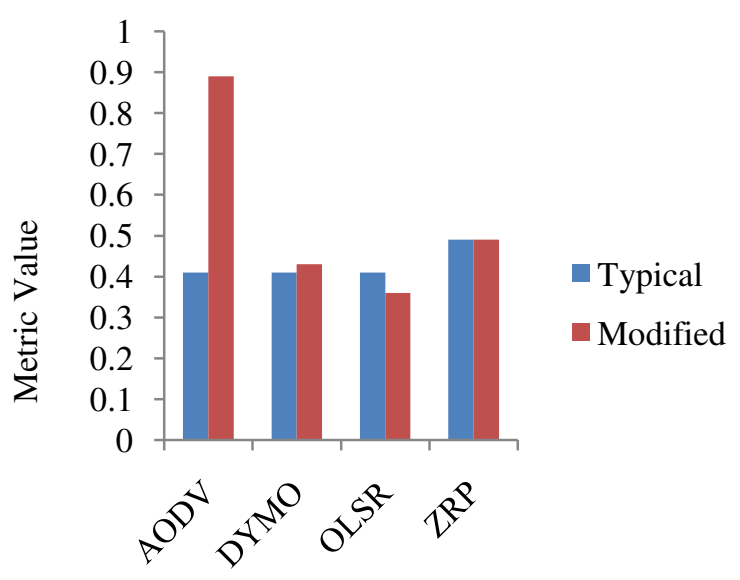

Fig. 4. No. of Data Request received.

\section{B. Signals Transmitted}

Fig. 5 shows the signals transmitted in WSN network. As it is clearly seen that in AAODV, ADYMO and AZRP more signals are transmitted as compared to AODV, DYMO and ZRP. But in AOLSR, fewer signals are transmitted as compared to OLSR.

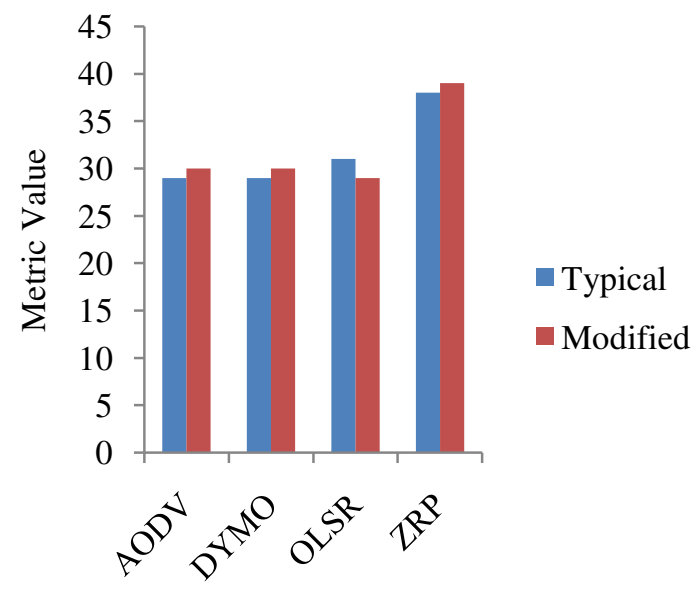

Fig. 5. Signals Transmitted.

\section{Signals Detected}

Fig. 6 shows signals detected in the network. It can be seen that typical AODV, DYMO, and ZRP have less signals detected in physical layer as compared to modified protocols. Whereas typical OLSR have detected less signals as compared to modified one. 


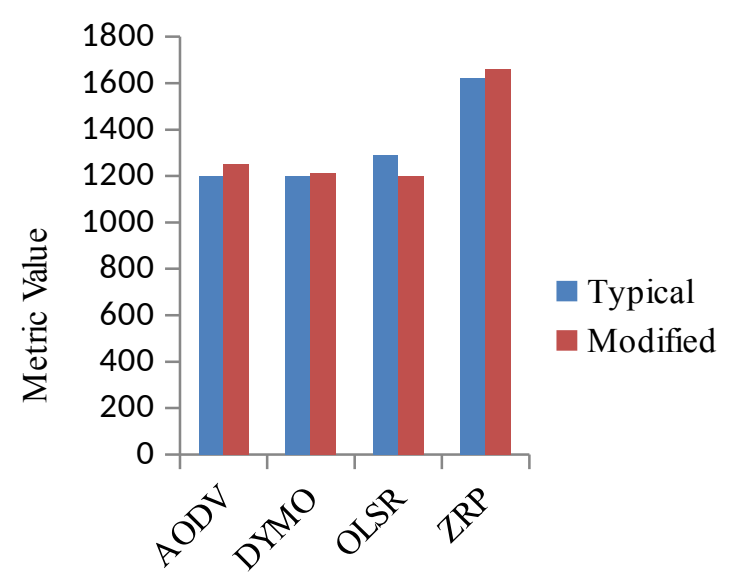

Fig 6: Signals Detected

\section{H. Number of data packets received}

Fig. 7 shows the number of data packets received in MAC layer. The value of packet received in AODV is 0.4 and AAODV is 3.9; value in DYMO is 0.6 and ADYMO is 0.4 ; value of OLSR is 4 whereas AOLSR is 2; value of ZRP is 15 and AZRP is 17. So, AAODV and AZRP shows more data packets received as compared to AODV and ZRP, whereas ADYMO and AOLSR receive fewer data packets as compared to DYMO and OLSR.

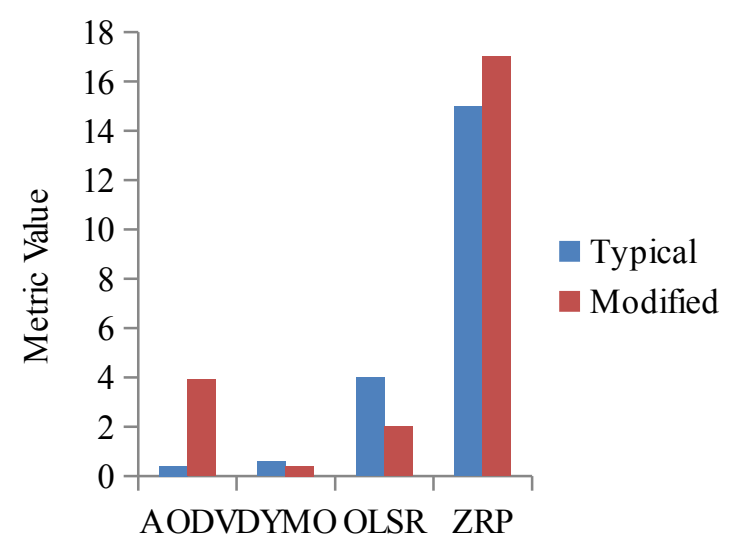

Fig 7: Number of data Packet Received

\section{Conclusion}

In this work, angle-based mechanism is used in routing protocols and then a comparison is done between typical and modified protocols are compared. Routing protocols used are AODV, DYMO, OLSR and ZRP, which are modified to
AAODV, AOLSR and AZRP. Results show that number of data request received is not much affected by using anglebased mechanism in protocols, except AAODV has received higher data requests as compared to AODV.

Signals transmitted have equally affected all the protocols, i.e. modified protocols transmit more signal as compared to typical protocols, except AOLSR, in which signal transmitted is decreased. Signals detected are also increased in all protocols except AOLSR in which fewer signals are detected. In AAODV and AZRP number of data packets received is increased as compared to AODV and ZRP respectively, whereas lesser number of data packets is received in ADYMO and AOLSR as compared to DYMO and OLSR. Number of packet dropped is also decreased in modified protocols, except AOLSR.

From all these results, it can be concluded that by adding angle-based mechanism, protocols have enhanced in many aspects. But it can be also seen that in every aspect modified OLSR does not responded well to the network. So, with respect to OLSR, this mechanism is not as much advantageous. All other protocols, AODV, DYMO and ZRP give approximately same changes by applying angle-based mechanisms. Therefore, this mechanism gives expected results for sensor networks. By using slight modifications in routing protocols, they can be used in sensor networks.

\section{REFERENCES}

[1] Nawaz, R., Hussain, S.A. ; Abid, S.A. ; Shafi, J.Beaconless multihop routing protocol for wireless sensor networks, in procedding of IEEE 3rd International Conference on Communication Software and Networks (ICCSN), pp: 721 - 725 DOI:10.1109/ICCSN. 2011.6014993 May, 2011.

[2] Mohammad A. Matin, "Wireless Sensor Networks Technology and Protocols"Publisher: Intech, September 2012

[3] K. Shim, K. Kim, S. Kim, "ADSR: Angle based Multi-hop Routing Strategy for Mobile WSNs" in IEEE Asia Pacific Services Computing Conference APSCC, pp: 373-376 DOI: 10.1109/APSCC.2011.27, 2011.

[4] Anne Jenefer and D. vydeki, "Performance Analysis of Mobile AdHoc Network in the presence of Wormhole attack" International Journal of Advanced Computer Engineering and Communication Technology (IJACECT), Volume:2, Issue:1, 2013, pp : 13-18.

[5] Anuj K. Gupta, JatinderKaur and SandeepKaur,"Comparison of DYMO, AODV, DSR and DSDV MANET routing protocols over varying traffic" IJREAS Volume 1, Issue 2 October, 2011.

[6] Thomas Heide Clausen, Gitte Hansen, Lars Christensen Gerd Behrmann, "The Optimized Link State Routing Protocol Evaluation through Experiments and Simulation" Mindpass Center for Distributed Systems Aalborg University, Fredrik Bajers Vej 7E, DK9220 Aalborg, Denmark.

[7] Nicklas Beijar "Zone Routing Protocol (ZRP)" Networking Laboratory, Helsinki University of Technology.

[8] Sandeep Kaur, Supreet Kaur, "Analysis Of Zone Routing Protocol In Manet" IJRET: International 12Journal of Research in Engineering and Technology, Volume: 02 Issue: 09, Sep-2013, pp: 520-524. 